\title{
THE EFFECTS OF SPATIO-TEMPORAL RESOLUTION ON DEDUCED SPICULE PROPERTIES
}

\author{
Tiago M. D. Pereira ${ }^{1,2}$, Bart De Pontieu ${ }^{2}$, and Mats Carlsson 3,4 \\ ${ }^{1}$ NASA Ames Research Center, Moffett Field, CA 94035, USA \\ ${ }^{2}$ Lockheed Martin Solar and Astrophysics Laboratory, 3251 Hanover Street, Org. A021S, Bldg. 252, Palo Alto, CA 94304, USA \\ ${ }^{3}$ Institute of Theoretical Astrophysics, P.O. Box 1029, Blindern, NO-0315 Oslo, Norway \\ ${ }^{4}$ Center of Mathematics for Applications, University of Oslo, P.O. Box 1053, Blindern, NO-0316 Oslo, Norway \\ Received 2012 October 30; accepted 2012 December 11; published 2013 January 28
}

\begin{abstract}
Spicules have been observed on the Sun for more than a century, typically in chromospheric lines such as H $\alpha$ and Ca II H. Recent work has shown that so-called "type II" spicules may have a role in providing mass to the corona and the solar wind. In chromospheric filtergrams these spicules are not seen to fall back down, and they are shorter lived and more dynamic than the spicules that have been classically reported in ground-based observations. Observations of type II spicules with Hinode show fundamentally different properties from what was previously measured. In earlier work we showed that these dynamic type II spicules are the most common type, a view that was not properly identified by early observations. The aim of this work is to investigate the effects of spatio-temporal resolution in the classical spicule measurements. Making use of Hinode data degraded to match the observing conditions of older ground-based studies, we measure the properties of spicules with a semi-automated algorithm. These results are then compared to measurements using the original Hinode data. We find that degrading the data has a significant effect on the measured properties of spicules. Most importantly, the results from the degraded data agree well with older studies (e.g., mean spicule duration more than 5 minutes, and upward apparent velocities of about $25 \mathrm{~km} \mathrm{~s}^{-1}$ ). These results illustrate how the combination of spicule superposition, low spatial resolution and cadence affect the measured properties of spicules, and that previous measurements can be misleading.
\end{abstract}

Key words: Sun: atmosphere - Sun: chromosphere - Sun: transition region

Online-only material: animations, color figure

\section{INTRODUCTION}

Spicules are dynamic structures in the solar chromosphere, first observed by Secchi (1877). Traditionally observed in $\mathrm{H} \alpha$, many of the earlier studies were reviewed by Beckers (1968, 1972). These reviews painted a classical view of spicules as jets ascending with apparent velocities around $25 \mathrm{~km} \mathrm{~s}^{-1}$, lifetimes of about 5 minutes, and reaching heights of 6-9 Mm. For several decades, this observational description has provided the constraints for spicule-formation mechanisms (Sterling 2000). The reported lifetimes of 5 minutes suggested a connection to the photospheric/granular timescales. The coronal heights reached made spicules candidates to transfer mass and energy from the photosphere to the corona (Beckers 1968; Pneuman \& Kopp 1978; Athay \& Holzer 1982; Tsiropoula \& Tziotziou 2004). Yet these enigmatic structures have eluded modelers partly because, as noted by Sterling (2000), older observations were not detailed enough and spicules were just beyond the resolution limits of instruments.

The launch of Hinode (Kosugi et al. 2007) and the combined use of adaptive optics and image post-processing (van Noort et al. 2005) in ground-based observations brought a breath of fresh air to the observational studies of spicules. By providing a seeing-free, stable observing platform with high spatial and temporal resolution, Hinode showed spicules in unprecedented detail. Using Hinode observations, De Pontieu et al. (2007) reported spicules as much more dynamic features than previously thought, and suggested the existence of two types of spicules: slower, longer-lived "type I" and faster, shorter-lived "type II." Recently, Pereira et al. (2012, hereafter Paper I) have extended this work by measuring a comprehensive set of spicules over several solar regions observed with Hinode. Paper I confirmed the existence of two types of spicules, finding that type I spicules to have typical lifetimes of $150-400 \mathrm{~s}$, ascending velocities of $15-40 \mathrm{~km} \mathrm{~s}^{-1}$, and type II spicules to have lifetimes of 50-150 s, and velocities of $30-130 \mathrm{~km} \mathrm{~s}^{-1}$. Besides the lifetimes and velocities, the major difference between type I and type II spicules is that the former rise and fall in a parabolic trajectory, while the latter only have a rise phase and then fade from the passband.

The fading of type II spicules has fueled the idea that they are rapidly heated to higher temperatures not visible in the Ca II $\mathrm{H}$ filter, and may deposit mass and energy at coronal heights (De Pontieu et al. 2007, 2009). Given the mass flux transported by spicules, even if only a few percent of the mass flux reaches coronal temperatures, spicules can help power the solar wind and energize the corona (Beckers 1968; Pneuman \& Kopp 1978; De Pontieu et al. 2009). This view of type II spicules being heated to coronal temperatures has been reinforced by De Pontieu et al. (2011), who find that some type II spicules, after fading from the $\mathrm{Ca}$ II $\mathrm{H}$ passband, appear in a transition region and coronal temperature filtergrams from the Atmospheric Imaging Array on board the Solar Dynamics Observatory. Thus, it is important to make the distinction between type I spicules (whose material falls back after it rises) and type II spicules, which have more potential to mediate the transfer of energy and mass from the chromosphere to the corona.

While type I spicules have properties consistent with the "classical" spicules of Beckers (1968, 1972), type II spicules do not. Therefore, the question arises: why are measurements of classical spicules seemingly incompatible with type II spicules seen from Hinode? The question is more pertinent as both De Pontieu et al. (2007) and Paper I find type I spicules abundant only in active regions, with Paper I finding type II spicules dominant in the quiet Sun and coronal holes. A plausible explanation is that the lower spatio-temporal resolution used before precluded the detection of the highly dynamic type II spicules. With 
Table 1

Observational Data Sets

\begin{tabular}{lcccccr}
\hline \hline Starting Time & $\begin{array}{c}x \text { Coord. } \\
(\operatorname{arcsec})\end{array}$ & $\begin{array}{c}y \text { Coord. } \\
(\operatorname{arcsec})\end{array}$ & $\begin{array}{c}\Delta t \\
(\mathrm{~s})\end{array}$ & $\begin{array}{c}\text { Duration } \\
(\text { minutes })\end{array}$ & $N_{\mathrm{O}}$ & $N_{\mathrm{D}}$ \\
\hline 2007 Apr 01T14:09 & 900 & -288 & 4.8 & 55 & 24 & 8 \\
2007 Apr 01T15:09 & 933 & -145 & 4.8 & 55 & 47 & 7 \\
2007 Aug 21T13:35 & 945 & 0 & 4.8 & 204 & 62 & 25 \\
2011 Sep 16T03:00 & -955 & 259 & 3.2 & 66 & 41 & 12 \\
\hline
\end{tabular}

Notes. $\Delta t$ is the observational cadence, $N_{\mathrm{O}}$ is the number of spicules detected in the original data, and $N_{\mathrm{D}}$ is the number of spicules detected in the degraded data.

such conditions, it is possible that spicules appearing and disappearing at the same place might be interpreted as one longerlived spicule, thereby biasing the measurements toward longer lifetimes and presumably different velocities. In this work, we put this hypothesis to the test by using the same spicule detection methods of Paper I on the same Hinode data but now degraded to approximate the conditions of previous studies. We then compare the results of spicules measured on the degraded data with those from Paper I. Our aim is to study the effects of the spatio-temporal resolution in spicule measurements, in particular those of early observations that provided the basis for the "classical" view on spicules.

\section{ANALYSIS AND RESULTS}

The data used are the quiet-Sun subset of Paper I. They span four sets of observations with Hinode/SOT Broadband Filter Imager (Tsuneta et al. 2008; Suematsu et al. 2008), in the Ca II H $(396.85 \mathrm{~nm})$ filter. These sets were chosen for their high cadence and quietness (e.g., absence of brightenings in X-ray images). The data were reduced using the standard IDL procedure fg_prep.pro, and co-aligned using the crosscorrelation technique of De Pontieu et al. (2007). A radial density filter (as in De Pontieu et al. 2007; Okamoto et al. 2007) was applied, along with a diagonal difference (emboss) filter to enhance the visibility of the spicules. The details of the observations are listed in Table 1.

To compare spicule measurements with previous studies we have degraded the Hinode data to mimic older observations. The spatial resolution has been degraded with a $1^{\prime \prime}$ FWHM Gaussian, and we sampled the images in time to get a cadence of $38.4 \mathrm{~s}$. The same radial density and emboss filters were applied to the degraded data. The effect of the spatial degradation is illustrated in Figure 1. Meant to encompass the heterogeneous range of parameters of older observations, these values are a simple approximation. Some studies had higher spatial resolution, others higher cadence (see Table 2), but overall we believe our values to be a reasonable approximation, in particular because other effects such as seeing were not introduced in the degradation. In this kind of filtergram analysis, seeing can have two main effects: a reduced spatial resolution from a consistent less than ideal seeing, and variable time series quality when the seeing varies significantly with time. With our data degradation, the spatial resolution effects of a constant seeing are accounted for, but not the time series effects. One should note that the effects of seeing on time series are probably often underestimated: only a few bad frames in a sequence with good seeing can be very disruptive for dynamic events such as spicules.

For our comparison, we use the quiet-Sun results of Paper I referring to them as "original," along with the results from

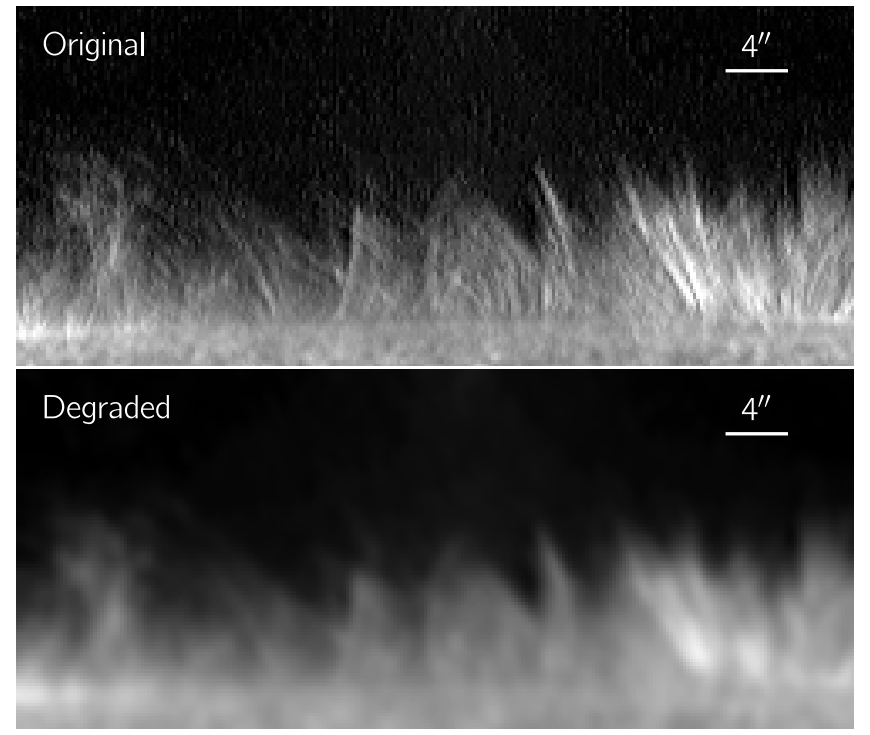

Figure 1. Sample images from 2007 August 21, 14:33:39 UT. The degraded image (bottom) was obtained by blurring the original image (top) with a Gaussian filter with a FWHM of $1^{\prime \prime}$. Radial density and emboss filters were applied to the images. Each panel is approximately $56^{\prime \prime} \times 24^{\prime \prime}$, and the approximate solar $(x, y)$ coordinates for the lower left corner are $\left(935^{\prime \prime} .9,28^{\prime \prime} .1\right)$. (An animation of this figure is available in the online journal.)

Table 2

Comparison of Mean Spicule Properties

\begin{tabular}{lcrccc}
\hline \hline Observations & $\begin{array}{c}\text { Cadence } \\
(\mathrm{s})\end{array}$ & $N$ & $\begin{array}{c}\langle t\rangle \\
(\mathrm{s})\end{array}$ & $\begin{array}{c}\left\langle v_{\text {up }}\right\rangle \\
\left(\mathrm{km} \mathrm{s}^{-1}\right)\end{array}$ & $\begin{array}{c}\left\langle H_{\max }\right\rangle \\
(\mathrm{Mm})\end{array}$ \\
\hline Roberts (1945) & 60 & 48 & 270 & 30 & 7.3 \\
Dizer (1952) & 30 & 353 & 132 & $20,40^{\mathrm{a}}$ & 7.4 \\
Rush \& Roberts (1954) & $10-60$ & 101 & 210 & 31 & 9.0 \\
Lippincott (1957) & $\approx 30$ & 77 & 342 & 24 & $7.3,9.5^{\mathrm{b}}$ \\
Nishikawa (1988) & $\approx 30$ & 4 & 339 & 42 & 8.7 \\
Christopoulou et al. (2001) & 24 & 4 & 353 & 43 & 4.9 \\
Pasachoff et al. (2009) & 49.2 & 40 & 426 & 27 & 7.2 \\
This work (original) & $3.2-4.8$ & 177 & 111 & 60 & 5.5 \\
This work (degraded) & 38.4 & $52^{\mathrm{c}}$ & 340 & 26 & 6.7 \\
\hline
\end{tabular}

Notes. Symbols denote the number of spicules $N$, lifetime $t$, apparent ascending velocity $v_{\text {up }}$, and maximum height $H_{\text {max }}$.

a The two values refer to "quiet" and "agitated" regions, respectively.

b The two values refer to equator and latitude $\pm 84^{\circ}$, respectively.

c Velocities were derived from 23 spicules.

the "degraded" data. For consistency, we used the same semiautomated algorithm, procedure, and settings of Paper I for detecting and measuring spicules. The method is briefly outlined below, with a more detailed description in Paper I.

The spicule detection is based on the algorithm used by Rouppe van der Voort et al. (2009) and tracks the time evolution of spicules, detecting spicules in each image and linking them with matching features in following images. From the detection algorithm, we obtain the coordinates of spicules linked across several images. These chains of events are visually inspected and purged of false positives (e.g., detections that are not spicules or spicules that are incorrectly linked). The next step is to measure the properties of spicules.

From the several quantities measured in Paper I, here we focus only on three: lifetime, maximum ascending velocity, and maximum height. The lifetime is obtained by visual inspection of the images before and after those in which a spicule was detected. The frames where the spicule is first and last seen are identified, and the lifetime is given by the time interval between 

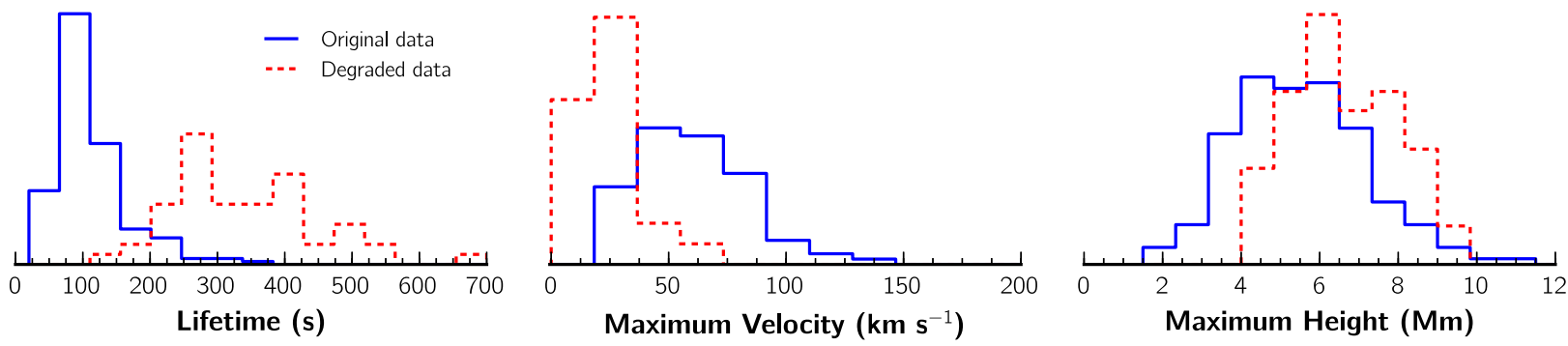

Figure 2. Normalized distributions of spicule properties for the original (blue solid) and degraded (red dashed) data.

(A color version of this figure is available in the online journal.)

them. Similarly, the maximum height is obtained by visual inspection of the images where the spicule is seen. The image where the spicule reaches its maximum height is identified and the height is measured as the closest distance from the top of the spicule to the limb. Finally, the maximum velocity is derived using space-time diagrams of the spicule intensity along its axis. Using the spicule coordinates provided by the algorithm, spacetime diagrams are built accounting for the spicular transverse motion. The approximate positions of the spicule tops for each time are identified in this diagram, and the velocity is extracted from a linear fit to these points.

Measuring limb spicules is a difficult task, which leads to a certain subjectivity in the measurements when the signal is not very clear. The large degree of spicule superposition at the limb is the main observational difficulty in tracking and measuring these objects. As discussed in Paper I, our spicule detection method has uncertainties and selection effects, but is nevertheless a consistent way to compare spicules across data sets and better than a purely visual estimation, in particular for comparing spicule properties in the original and degraded data.

In the degraded data we detected a total of 52 spicules. Of these, we derived the velocities from only 23 spicules, which provided the clearest signal. This is perhaps too conservative, but we wanted to keep our sample as clear as possible from less accurate measurements. From Table 1 one can see that in the original data over three times more spicules were detected. This discrepancy comes largely from the spatial degradation, whose blurring lowers the contrast of spicules and makes the automated detection harder. In Paper I more than $98 \%$ of the spicules in these observations were found to be of type II, and the remaining of type I. In the smaller numbers of spicules detected in the degraded data, we did not find spicules with a parabolic rise and fall motion (as type I spicules), or spicules with a descending phase.

The distributions of the measured spicule properties are shown in Figure 2. For the degraded data we obtain a mean lifetime of $340 \mathrm{~s}$ with a standard deviation of $\sigma=107 \mathrm{~s}$; a mean maximum velocity of $25.8 \mathrm{~km} \mathrm{~s}^{-1}$ with $\sigma=11.4 \mathrm{~km} \mathrm{~s}^{-1}$; and a mean maximum height of $6.66 \mathrm{Mm}$ with $\sigma=1.35 \mathrm{Mm}$. For the original data the results were a mean lifetime of $111 \mathrm{~s}$ with $\sigma=54 \mathrm{~s}$; a mean maximum velocity of $60.0 \mathrm{~km} \mathrm{~s}^{-1}$ with $\sigma=23.3 \mathrm{~km} \mathrm{~s}^{-1}$; and a mean maximum height of $5.51 \mathrm{Mm}$ with $\sigma=1.70 \mathrm{Mm}$.

\section{DISCUSSION}

\subsection{Degraded Data and Classical Spicules}

Measuring spicules from degraded Hinode data has a dramatic effect on their properties, as evidenced by Figure 2. Even more striking, the values from the degraded data agree very well with the classical results for spicules. It can be argued that our analysis suffers from systematic effects and uncertainties (like all studies of spicules, but which we minimize here as much as possible). However, the differential analysis between original and degraded data cancels those systematics and undeniably proves the effects of data degradation on spicule properties. Our results are not meant to provide the universal values of spicules measured from degraded data - the rather approximate nature of our degradation precludes it—but instead illustrate how such degradation can affect measurements of spicules.

In Table 2 we compare our results with those of several ground-based studies, such as Roberts (1945), Dizer (1952), Rush \& Roberts (1954), and Lippincott (1957), which provided the basis for the reviews of Beckers $(1968,1972)$. Additionally, we include the results of Nishikawa (1988) and Christopoulou et al. (2001). The more recent work of Pasachoff et al. (2009) is also included, even though the authors used a modern telescope (Swedish Solar Telescope; SST; Scharmer et al. 2003) with higher spatial resolution than Hinode/SOT. Nevertheless, the authors used a very long cadence of $49.2 \mathrm{~s}$ and despite the high spatial resolution the observations were still subject to seeing effects in the time series.

All of the ground-based observations we compare with were made with $\mathrm{H} \alpha$ filters, while the Hinode data were observed in the $\mathrm{Ca}$ II $\mathrm{H}$ filter. We are making the assumption that limb spicules should be comparable with both diagnostics. Spectroscopic studies show that $\mathrm{H} \alpha$ and $\mathrm{Ca} I \mathrm{H}$ and $\mathrm{K}$ have similar Doppler velocities (Athay \& Bessey 1964; Alissandrakis 1973). Imaging studies such as the early work of Athay \& Roberts (1955) and most recently McIntosh et al. (2008) show similar limb structures in $\mathrm{H} \alpha$ and $\mathrm{Ca}$ II filters. We used the same Hinode SOT/NFI data as McIntosh et al. (2008) to construct $\mathrm{H} \alpha$ filtergrams and compare them with simultaneous SOT/BFI Ca II $\mathrm{H}$ filtergrams. In Figure 3 we compare both filtergrams. These observations were taken starting at 14:46 UT on 2007 September 27. The $\mathrm{H} \alpha$ filtergrams shown were obtained by summing NFI filtergrams at $\mathrm{H} \alpha \pm 80 \mathrm{pm}$ (red and blue wings, observed $3.2 \mathrm{~s}$ apart). It is clear from Figure 3 and the animation that both the spicule structure and the time evolution are very similar in $\mathrm{H} \alpha$ and $\mathrm{Ca}$ II $\mathrm{H}$. Thus, it seems reasonable to compare measurements from both diagnostics.

Many older studies do not distinguish between quiet Sun, coronal holes, or active regions. With observations spanning a large area of the Sun, the spicules studied were a sample of many regions. For our comparison, we focused on quiet-Sun observations because the properties of quiet Sun and coronal hole spicules are very similar (Paper I) and in the degraded data the difference would be negligible. Active regions were not included because they are not as common and were not singled out in the description of classical spicules.

With typical cadences between 30 and 60 s, older groundbased observations could only observe type II spicules in a few 

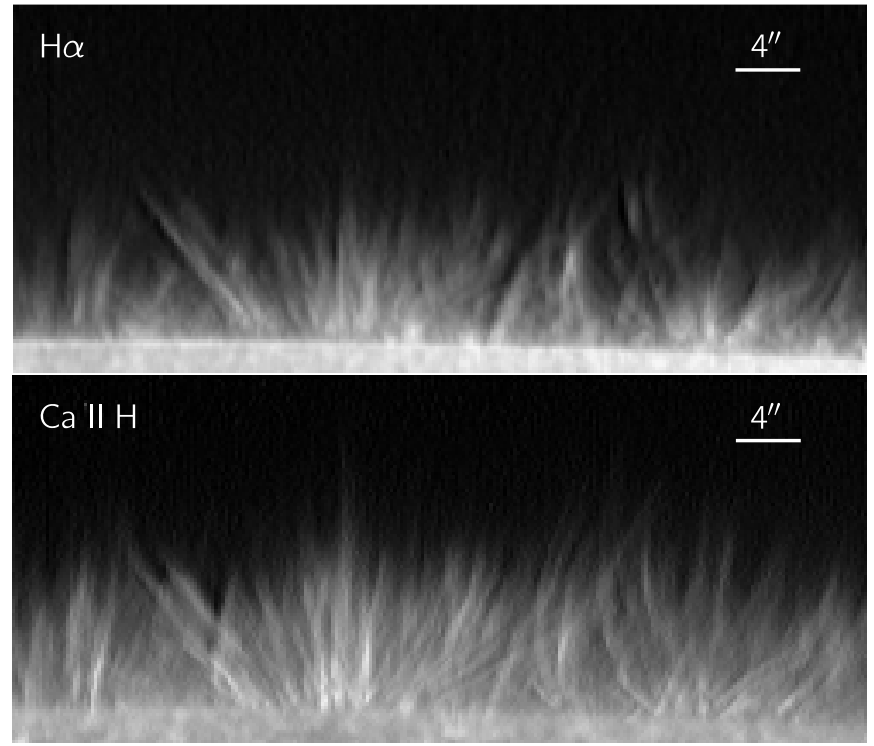

Figure 3. $\mathrm{H} \alpha$ and $\mathrm{Ca}$ II $\mathrm{H}$ filtergrams from SOT polar observations taken at 14:46:32 UT on 2007 September 27. H $\alpha$ filtergram is the sum of red and blue wing NFI filtergrams $(\mathrm{H} \alpha \pm 80 \mathrm{pm}$, taken $3.2 \mathrm{~s}$ apart $)$. Each Ca II H BFI filtergram was observed $1.6 \mathrm{~s}$ after the second $\mathrm{H} \alpha$ exposure. Radial density and emboss filters were applied to the images. Each panel is approximately $56^{\prime \prime} \times 24^{\prime \prime}$, and the approximate solar $(x, y)$ coordinates for the lower left corner are $\left(-2^{\prime \prime} 4,951^{\prime \prime} .9\right)$.

(An animation of this figure is available in the online journal.)

frames. It is perhaps not surprising that spicules with typical lifetimes of 50-150 s (Paper I) were generally not found. With the exception of Dizer (1952), all of the listed studies give mean lifetimes above $200 \mathrm{~s}$. In terms of mean ascending velocities, the first four studies in Table 2 have established the Beckers canonical value of $25 \mathrm{~km} \mathrm{~s}^{-1}$. At the time, this value was accepted as representative also because it was consistent with the Doppler velocities derived from spectroscopic observations (Beckers 1968). Nishikawa (1988) and Christopoulou et al. (2001) find higher velocities, but from only four spicules each, all of which had parabolic trajectories (suggesting type I and not type II spicules).

Using higher spatial resolution observations, Pasachoff et al. (2009) obtain results that agree very well with the classical values. While enormous progress has been made in groundbased observations on the solar disk with adaptive optics and image reconstruction techniques (van Noort et al. 2005), the effects of seeing are harder to correct in limb images because of the lack of structures from which to derive the wavefront distortions. Given the long cadence used and the difficulties in correcting for seeing at the limb, it is perhaps not surprising that the measurements of Pasachoff et al. (2009) are consistent with classical spicules.

Zhang et al. (2012) claim that type II spicules may not even exist. Using Hinode $\mathrm{Ca}$ II $\mathrm{H}$ filtergrams, the authors measure spicule properties and find most to be similar to type I spicules and thus compatible with the classical spicules. We disagree with their conclusions. In Paper I we analyze the same data sets and find a dominance of type II spicules, and inconsistencies within their measurements. With the present work we show that the existence of type II spicules is compatible with the measurements of classical spicules, given the lower resolution data used in the past.

After their rise phase, classical spicules have been reported to either fade or descend. The fraction of the spicules that descend (instead of fading) is not always reported, but can be more than half (e.g., Lippincott 1957 finds descent in 60\% of spicules, Pasachoff et al. 2009 only $28 \%$ ). In most of these cases the descent motions were irregular; Beckers (1968) notes that several studies "notice the descent to be much more poorly defined than the ascent." Rush \& Roberts (1954) note that the descent was often "abrupt and irregular, suggesting that an apparent descent was actually a progressive loss of visibility." This hints that at least some of the descents of spicules could be artifacts caused by seeing and low resolution; a "loss of visibility" is more consistent with the fading associated with type II spicules. In our original data analysis on Paper I, we find only $2 \%$ of descending spicules in these data. In the degraded data we find no descending spicules, possibly owing to the lower number of detected spicules. This is at odds with the earlier reports. The reasons for this discrepancy are unclear. Some of the apparently descending spicules observed from the ground could be an artifact of seeing and resolution (as suggested by Rush \& Roberts 1954). It is also possible that these descents are less visible in $\mathrm{Ca}$ II $\mathrm{H}$ than in $\mathrm{H} \alpha$ because of the different passbands.

Both Nishikawa (1988) and Christopoulou et al. (2001) find up and down parabolic motions in all four spicules measured, which suggests that they are type I spicules. At least for the higher-quality observations of Christopoulou et al. (2001), it seems unlikely that the detection of parabolic motion results from data degradation. While Paper I finds a scarcity of type I spicules in quiet Sun and coronal hole regions, it is unclear if there are nearby active regions in the observations of Nishikawa (1988) and Christopoulou et al. (2001). Christopoulou et al. (2001) find a connection between observed spicules and mottles, further suggesting that the spicules studied are of type I, whose properties are consistent with classical spicules (see the discussion on type I spicules and mottles in Paper I).

\subsection{How Does Data Degradation Affect Spicule Measurements?}

Having established how the data degradation affect the measurements, it is important to investigate why. Looking at the degraded data and following the detected spicules, we identify several trends that affect the measurements. The clearest of those is what makes lifetimes longer. With a lower cadence and spatial resolution, following spicules is made difficult by their dynamic nature. It often happens that spicules recur from similar footpoints, clustered in a few regions, and with a high degree of superposition (Paper I). Taking their transverse motions into account, when observed with long cadences it is often difficult to identify the same spicules in the next image. Adding a lower spatial resolution and many of these nearby spicules merge into each other, becoming extremely difficult to disentangle. Thus, the automated detection (and human eye) is fooled into thinking that a longer event is the same spicule, when in reality a much more dynamic scenario of spicules coming and going, and moving transversely occurs. Because now these spicules are measured over longer lifetimes, the likelihood of reaching larger heights increases because by bundling several spicules together we will measure the tallest in that group. This shifts the height distribution to larger values (but does not affect the largest values of the distribution, as should be expected). The effect on the apparent velocities is more convoluted and not so clear cut. A possible explanation is that with longer cadences one tends to miss the initial rise phase of the spicules, which represents a smaller fraction of their lifetime. Hence, one 
would preferentially sample a smaller range of spicule heights that together with the longer lifetimes would yield a lower apparent velocity. In such conditions one should also expect to get several irregular space-time diagrams for the spicule lengths, where no clear ascent or descent of the spicule is visible. This is corroborated by the fact that we could not get a reliable measurement of velocity from about half of the detected spicules because of such irregular diagrams. This was often also the case in classical studies, where several authors only derived velocities from a small fraction of the spicules used for height measurements (Rush \& Roberts 1954; Lippincott 1957; Nishikawa 1988).

It is likely that lifetimes derived from classical studies of spicules are instead related to other dynamic timescales, such as spicule recurrence. It has been noted that spicules recur from the same footpoints (Beckers 1968; Paper I). Using transition region and coronal lines, McIntosh \& De Pontieu (2009) analyzed upflows believed to be associated with type II spicules and find that these upflows recur with a timescale around 3-15 minutes. This range is consistent with the lifetimes derived from classical spicules, suggesting that those lifetimes reflect not the lifetime of an individual spicule, but the lifetime of a larger ensemble: the timescale of spicule recurrence. Sekse et al. (2013) find similar recurrence of Rapid Blueshifted Events, the likely diskcounterpart of type II spicules.

In this view, the classical features are actually conglomerates of ("type II") spicules. This is compatible with the speculation by Zaqarashvili et al. (2007) of "micro-spicules" constituting the coarser spicules detected with their high cadence and lowresolution coronagraphic observations.

\section{CONCLUSIONS}

The recent discovery of two types of spicules (De Pontieu et al. 2007) shows that spicules are more dynamic than previously thought. Type II spicules in particular have short lifetimes (50-150 s), high ascending velocities $\left(30-130 \mathrm{~km} \mathrm{~s}^{-1}\right)$, and are dominant in most of the solar limb, except in active regions (Paper I).

The properties of type II spicules (De Pontieu et al. 2007; Paper I) are incompatible with the classical views on spicules, consisting of lifetimes around 5 minutes and apparent velocities of $25 \mathrm{~km} \mathrm{~s}^{-1}$ (Beckers 1968, 1972). Because type II spicules are likely to dominate in most locations where classical spicules were observed, it becomes a problem to reconcile both views. We suggest that these differences arise from a mix of spicule superposition and the low spatial and temporal resolution of older observations. To test this hypothesis, we degrade Hinode/SOT observations to similar resolution and cadence of older studies, and analyze the spicules using the same method as Paper I. Comparing the results of the original against the degraded data, we find that the image degradation has a significant effect on the measured spicule properties. For the degraded data we derive a mean lifetime around 5 minutes and a mean ascending velocity of $26 \mathrm{~km} \mathrm{~s}^{-1}$, in very good agreement with the classical values.

We conclude that type II spicules were likely previously detected in the studies reviewed by Beckers, but that their low spatial and temporal resolutions did not allow for an accurate determination of their dynamic properties. This resolves the puzzling discrepancy between spicules seen from Hinode and older ground-based studies, and firmly establishes that the classical properties are not representative. Theoretical studies of spicule formation, which so far have relied mostly on the classical spicule description, cannot afford to ignore the fundamentally different properties of type II spicules. These must be taken into account to provide model constraints.

T.M.D.P. was supported by the NASA Postdoctoral Program at Ames Research Center (NNH06CC03B). B.D.P. was supported by NASA (NNX08AH45G, NNX08BA99G, and NNX11AN98G). This research was supported by the Research Council of Norway. The research leading to these results has received funding from the European Research Council under the European Union's Seventh Framework Programme (FP7/20072013)/ERC Grant agreement 291058. Hinode is a Japanese mission developed by ISAS/JAXA, with the NAOJ as domestic partner and NASA and STFC (UK) as international partners. It is operated in cooperation with ESA and NSC (Norway). We thank Silje Bjølseth and Anne Fox for help with data reduction.

\section{REFERENCES}

Alissandrakis, C. E. 1973, SoPh, 32, 345

Athay, R. G., \& Bessey, R. J. 1964, ApJ, 140, 1174

Athay, R. G., \& Holzer, T. E. 1982, ApJ, 255, 743

Athay, R. G., \& Roberts, W. O. 1955, ApJ, 121, 231

Beckers, J. M. 1968, SoPh, 3, 367

Beckers, J. M. 1972, ARA\&A, 10, 73

Christopoulou, E. B., Georgakilas, A. A., \& Koutchmy, S. 2001, SoPh, 199, 61 De Pontieu, B., McIntosh, S. W., Carlsson, M., et al. 2011, Sci, 331, 55

De Pontieu, B., McIntosh, S. W., Hansteen, V. H., \& Schrijver, C. J. 2009, ApJL, 701, L1

De Pontieu, B., McIntosh, S., Hansteen, V. H., et al. 2007, PASJ, 59, 655

Dizer, M. 1952, CRAS, 235, 1016

Kosugi, T., Matsuzaki, K., Sakao, T., et al. 2007, SoPh, 243, 3

Lippincott, S. L. 1957, SCoA, 2, 15

McIntosh, S. W., \& De Pontieu, B. 2009, ApJ, 707, 524

McIntosh, S. W., De Pontieu, B., \& Tarbell, T. D. 2008, ApJL, 673, L219

Nishikawa, T. 1988, PASJ, 40, 613

Okamoto, T. J., Tsuneta, S., Berger, T. E., et al. 2007, Sci, 318, 1577

Pasachoff, J. M., Jacobson, W. A., \& Sterling, A. C. 2009, SoPh, 260, 59

Pereira, T. M. D., De Pontieu, B., \& Carlsson, M. 2012, ApJ, 759, 18

Pneuman, G. W., \& Kopp, R. A. 1978, SoPh, 57, 49

Roberts, W. O. 1945, ApJ, 101, 136

Rouppe van der Voort, L., Leenaarts, J., de Pontieu, B., Carlsson, M., \& Vissers, G. 2009, ApJ, 705, 272

Rush, J. H., \& Roberts, W. O. 1954, AuJPh, 7, 230

Scharmer, G. B., Bjelksjo, K., Korhonen, T. K., Lindberg, B., \& Petterson, B. 2003, Proc. SPIE, 485, 341

Secchi, A. 1877, Le Soleil, Vol. 2 (Paris: Gauthier-Villars)

Sekse, D. H., Rouppe van der Voort, L., \& De Pontieu, B. 2013, ApJ, in press (arXiv:1212.4988)

Sterling, A. C. 2000, SoPh, 196, 79

Suematsu, Y., Tsuneta, S., Ichimoto, K., et al. 2008, SoPh, 249, 197

Tsiropoula, G., \& Tziotziou, K. 2004, A\&A, 424, 279

Tsuneta, S., Ichimoto, K., Katsukawa, Y., et al. 2008, SoPh, 249, 167

van Noort, M., Rouppe van der Voort, L., \& Löfdahl, M. G. 2005, SoPh, 228,191

Zaqarashvili, T. V., Khutsishvili, E., Kukhianidze, V., \& Ramishvili, G. 2007, A\&A, 474, 627

Zhang, Y. Z., Shibata, K., Wang, J. X., et al. 2012, ApJ, 750, 16 